\title{
INVESTIGAÇÃO DE NEUROPATIA PERIFÉRICA DURANTE 0 PERÍODO RECENTE DO TRANSPLANTE DE MEDULA ÓSSEA
}

\author{
VIVIANE H. FLUMIGNAN ZÉTOLA*, ROSANA HERMÍNIA SCOLA**, \\ RICARDO PASQUINI***, LINEU CESAR WERNECK ${ }^{* * *}$
}

\begin{abstract}
RESUMO - Investigamos prospectivamente a incidência de neuropatia periférica em 43 pacientes através do estudo da velocidade de condução nervosa e do teste de limiar de sensibilidade vibratória (palestesiômetro) realizados antes e após o transplante de medula óssea. Nesse período as principais drogas utilizadas para o condicionamento e imunossupressão foram o bussulfan, ciclofosfamida, ciclosporina A, methotrexate e corticoesteróides. Foram estudadas as velocidades de condução nervosa nos nervos mediano motor, fibular, tibial, mediano sensitivo e sural. Obtivemos alterações estatisticamente significativas na duração do potencial composto proximal do nervo mediano motor, na amplitude distal do nervo tibial posterior e na amplitude proximal do nervo sural. As diferenças observadas não se correlacionaram com alterações clínicas, e não foram suficientes para o diagnóstico de neuropatia periférica. Acreditamos que o esquema terapêutico utilizado não provoca toxicidade neurológica periférica no período recente do transplante de medula óssea.
\end{abstract}

PALAVRAS-CHAVE: neuropatia periférica, estudo de condução nervosa, transplante de medula óssea.

\section{Peripheral neuropathy investigation in the early stage of bone marrow transplantation}

ABSTRACT - Forty-three patients with several hematological diseases in the early stage of bone marrow transplantation were prospectively studied. All patients underwent a complete neurological examination, vibratory sense perception test and nerve conduction study both before and after the bone marrow transplantation. The following nerves were studied: median, peroneal, posterior tibial (motor), median and sural (sensitive). Most patients were in busulphan, cyclophosphamide, cyclosporine A, methotrexate and corticosteroids. Although the results showed statistical differences in the nerve conduction study, all of these differences were not sufficient to induce or diagnose peripheral nerve injury. Therefore, it was concluded that drugs used in the bone marrow transplantation do not induce peripheral neuropathy.

KEY WORDS: peripheral neuropathy, nerve conduction study, bone marrow transplantation.

O transplante de medula óssea (TMO) é considerado, atualmente, o segundo transplante de órgão mais frequente, sendo tratamento de escolha para uma série de doenças malignas hematológicas, não-hematológicas, genéticas e imunológicas ${ }^{1-3}$. O controle e manejo de suas complicações é objeto de muitos estudos que buscam evitar a toxicidade das drogas, a rejeição e falência do enxerto, a doença enxerto contra hospedeiro e as infecções oportunistas. As complicações neurológicas ainda são frequentes e causam óbito em aproximadamente 6-18\% dos $\operatorname{casos}^{4-7}$.

O sistema nervoso periférico é o menos acometido ${ }^{4-7}$, e não tem sido objeto de estudo. A incidência de drogas induzindo lesões de nervos periféricos é de difícil estabelecimento. Os avanços

Serviço de Doenças Neuromusculares, Especialidade de Neurologia e Hematologia do Departamento de Clínica Médica do Hospital de Clínicas (HC) da Universidade Federal do Paraná (UFPR), Curitiba, Brasil: *Neurologista; **Professora Assistente; ***Professor Titular. Aceite: 27-fevereiro-1998.

Dra. Viviane Flumignan Zétola - Serviço de Doenças Neuromusculares, Hospital de Clínicas - Rua General Carneiro 181, $3^{\circ}$ andar - 80060-900 Curitiba PR - Brasil. 
nesse entendimento são limitados pelo potencial de reversibilidade da maioria dessas desordens, o que diminui as oportunidades para o estudo fisiológico e histológico ${ }^{8,9}$. Esquemas terapêuticos anteriores foram substituídos ou tiveram o seu uso restrito devido aos efeitos colaterias nos nervos periféricos (cisplatina, vincristina, citarabina e talidomida) ${ }^{8,9}$. As combinações atuais não têm sido reportadas como lesivas, no entanto alguns autores sugerem que com o uso preferencial da politerapia, terapias em altas doses e terapias experimentais, a incidência de neuropatia periférica seguida ao uso de drogas continua aumentando, podendo traduzir um efeito aditivo ou sinérgico ${ }^{10-12}$. Outras desordens devem ser consideradas nesses pacientes submetidos ao TMO como as de origem carencial, séptica ou associada a falência de múltiplos órgãos que podem contribuir ou mesmo originar quadro neuropático nesses indivíduos ${ }^{13-15}$.

O Serviço de Hematologia do Departamento de Clínica Médica do Hospital de Clínicas da UFPR presta atendimento a aproximadamente 100 pacientes por ano acometidos por doenças cujo potencial terapêutico reside no TMO. Tendo em vista esta larga amostragem, objetivamos avaliar prospectivamente o envolvimento do nervo periférico durante o período recente pós-TMO.

\section{MATERIAL E MÉTODOS}

Realizado estudo observacional (transversal) e prospectivo de 43 pacientes, no período de novembro1993 a maio-1995. Os critérios de inclusão foram :1. Primeiro transplante (alogênico ou autólogo); 2. Doenças de base: leucemias, anemias aplástica e mielodisplasia; 3. Não ter iniciado o regime de condicionamento antes da primeira avaliação; 4. Primeiro exame de condução nervosa normal.

O tempo de diagnóstico da doença de base variou de 0,08 anos a 6 anos (média 1,33 anos). A maioria dos pacientes possuía tratamentos prévios ao transplante, que incluíam quimioterapia em 26 (32,6\%), transfusões sanguíneas em $11(25,6 \%)$ e ambos em $10(23,3 \%)$. A distribuição por sexo foi $15(34,9 \%)$ mulheres e 28 $(65,1 \%)$ homens. A idade variou entre 12 e 53 anos, sendo a média de 28,58 anos $\pm 10,15$. As doenças de base que motivaram a realização do transplante foram assim distribuídas: $16(37,2 \%)$ leucemia mielóide crônica; 15 $(34,9 \%)$ anemia aplástica severa; 8 (18,6\%) leucemia mielóide aguda; 3 (7\%) mielodisplasia; e 1 (2,3\%) leucemia linfocítica aguda. $\mathrm{O}$ condicionamento (período destinado à ablação da medula óssea do receptor e à inibição da sua imunidade), bem como o regime de imunossupressão, variaram de acordo com a doença de base para o transplante, sendo que todos os pacientes usaram ciclofosfamida (CFA), 40 (93\%) a combinação com bussulfan (BUS) e ciclosporina(CSA), 36 (83,70\%) com methotrexate (MTX) e 18 (41,95\%) com corticoesteróides (CTC). Ainda como protocolo do serviço, todos os pacientes utilizaram sulfametoxazol + trimetoprima para a profilaxia do Pneumocisti carini, aciclovir para a profilaxia do Herpes vírus e o norfloxacino visando à esterilização do cólon. Não foram utilizadas drogas por via intratecal nem realizada irradiação corporal total nesse período.

Os pacientes foram submetidos a um protocolo de anamnese dirigida, exames segmentar e neurológico (padrão), ao teste do limiar da percepção vibratória (LPV) e ao estudo da velocidade de condução nervosa (VCN). A anamnese inicial constou de questões direcionadas à detecção de fatores de risco predisponentes de lesões periféricas como a exposição a agrotóxicos ou químicos industriais, uso de drogas, abuso do álcool, doenças associadas, história familiar para distúrbios neuromusculares, atividade profissional e traumatismos. O exame neurológico constou de testes de função cognitiva (orientação tempo/espaço, memória, cálculos e pensamento abstrato, pesquisa dos nervos cranianos, avaliação motora - enfatizando musculatura distal dos pés, avaliação da sensibilidade superficial (dor, tacto fino) e profunda (posição segmentar, equilíbrio com olhos abertos e fechados), pesquisa de reflexos superficiais e profundos, marcha, coordenação, palpação de nervo periférico e manobras de detecção de irritação meníngea. Todos os exames de VCN foram realizados no eletromiógrafo modelo Nihon Kohden Neuropack 2 seguindo-se as técnicas de exame utilizadas no Serviço de Doenças Neuromusculares do HC da UFPR ${ }^{16}$. Examinamos, através de eletrodos cutâneos de superfície, os nervos mediano motor, fibular profundo, tibial posterior e mediano sensitivo e sural direitos. O limiar de percepção vibratória (LPV) foi medido com o auxílio do palestesiômetro - "Bio Thesiometer", modelo PVD (Bio Medical Instrument Co., Newbury, Ohio, USA). As áreas selecionadas para a medida do LPV foram a porção dorsal da primeira falange dos $2^{\circ}$ e $5^{\circ}$ quirodáctilos direitos e o dorso do $1^{\circ}$ e $5^{\circ}$ pododáctilos direitos. Os valores foram calculados pela média aritmética de 10 testes $^{17,18}$. Tanto o estudo da VCN quanto o LPV foram realizados nos períodos admissional imediato e pós-transplante, na ocasião da alta hospitalar (média de 35.41 dias - mínimo de 22 e máximo de 90 dias). Excepcionalmente alguns pacientes foram submetidos a uma avaliação extra neste período. 
O protocolo do Serviço de TMO do HC da UFPR realiza seguimentos quase diários de hemograma completo, contagem de plaquetas, glicemia, sódio, potássio, magnésio, creatinina, proteínas séricas e testes de função hepática, entre outros. Solicitamos a inclusão das dosagens séricas de creatinoquinase (CK), aldolase e provas de atividades inflamatórias, que foram realizadas semanalmente durante o período de internação.

Os resultados dos testes e exames foram submetidos a tratamento estatístico, que consistiu na aplicação do teste t de "Student". Para todas as análises, as diferenças foram consideradas significativas no nível de 5\% (p $<0,05)$.

\section{RESULTADOS}

Em relação aos fatores de risco para o desenvolvimento da neuropatia periférica, a principal associação clínica foi o uso de inseticidas, que ocorreu em três $(7,0 \%)$ dos pacientes (Tabela 1). A dificuldade de marcha em $12(27,9 \%)$ e a fraqueza generalizada em $11(25,65 \%)$ foram as principais queixas envolvendo o sistema nervoso periférico relatadas durante a anamnese dirigida. Não houve outras alterações sintomáticas no decorrer da internação. Quanto ao exame físico segmentar, a principal alteração observada foi a mucosite, em 33 (76,7\%) dos pacientes na fase pós-TMO. A dificuldade de deglutição e a presença de sialorréia foram observadas nestes pacientes e consideradas secundárias. O exame neurológico apresentou como principais alterações distúrbios da coordenação motora e dos reflexos profundos na fase pós-TMO (Tabela 2). As diferenças não foram estatisticamente significantes.

Obtivemos diferenças significativas na análise estatística dos níveis séricos de glicose e albumina (Tabela 3). O uso do palestesiômetro na avaliação do limiar de excitabilidade vibratória não demonstrou alterações significativas quando os resultados foram comparados ao primeiro exame de controle. A análise do VCN para os nervos fibular e mediano sensitivo foram normais. Obtivemos aumento na duração do potencial composto proximal do nervo mediano motor, diminuição da amplitude distal do nervo tibial posterior e aumento da amplitude proximal do nervo sural, com níveis de significância entre 0,01 e 0,05, considerando o universo dos pacientes (Tabela 4).

Os dados também foram analisados de acordo com o uso de medicações nesse período. Trinta e quatro pacientes - o número mais expressivo de nossa amostra - utilizaram a combinação de BUS, CFA, MTX e CSA. Nesses, também não foram detectadas diferenças significativas no estudo dos nervos fibular e mediano sensitivo. Já, as alterações obtidas no aumento da duração do potencial composto proximal do nervo mediano motor e na diminuição da amplitude proximal do nervo sural foram significativas. A diferença da amplitude distal do nervo tibial nesse grupo não correspondeu à casuística total. A duração do potencial composto proximal do nervo mediano motor apresentou significância $\mathrm{p}=0,003$ e a amplitude proximal do nervo sural $\mathrm{p}=0,0381$ na análise dos 34 casos selecionados.

Tabela 1. Fatores predisponentes para neuropatia periférica.

\begin{tabular}{lc}
\hline Fatores associados & Frequência \\
\hline Uso de inseticidas/agrotóxicos & $3(7,0 \%)$ \\
Tto de hipertensão arterial sistêmica & $2(4,7 \%)$ \\
Tto de epilepsia & $2(4,7 \%)$ \\
Tto de doenças reumatológicas & $1(2,3 \%)$ \\
Tto de tuberculose & $1(2.3 \%)$ \\
Tto de hanseníase & $1(2,3 \%)$ \\
Uso de drogas recreativas* & $1(2,3 \%)$ \\
Insuficiência renal & $1(2,3 \%)$ \\
\hline
\end{tabular}

Tto, tratamento; *maconha e cocaína. 
Tabela 2. Alterações do exame neurológico.

\begin{tabular}{lc}
\hline Alterações & Frequência \\
\hline Hiperreflexia patelar & $11(25,6 \%)$ \\
Hiperreflexia aquiliana & $7(16,3 \%)$ \\
Tremor de repouso e ação + coréia & $3(7,0 \%)$ \\
Tremor de repouso, ação e postural & $3(7,0 \%)$ \\
Tremor de ação & $1(2,3 \%)$ \\
Dismetria + disdiadococinesia & $1(2,3 \%)$ \\
Coréia & $1(2,3 \%)$ \\
\hline
\end{tabular}

Tabela 3. Índices significativos na análise sérica dos exames laboratoriais.

\begin{tabular}{lccc}
\hline Níveis & Pré-TMO & Pós-TMO & Significância \\
\hline Glicemia (mg/dl) & $102,9 \pm 20,1$ & $114,8 \pm 32,8$ & $\mathrm{p}=0,046$ \\
Albuminemia (mg/dl) & $3,77 \pm 0,70$ & $3,28 \pm 0,36$ & $\mathrm{p}=0,0001$ \\
\hline
\end{tabular}

Tabela 4. Análise da velocidade da condução nervosa (metro/segundo).

\begin{tabular}{|c|c|c|c|}
\hline VCN MOTORA & Pré-Transplante & Pós-Transplante & Significância \\
\hline \multicolumn{4}{|l|}{ Mediano } \\
\hline Latência distal & $3,07 \pm 0,36$ & $3,20 \pm 0,79$ & NS \\
\hline Latência proximal & $7,33 \pm 0,78$ & $7,30 \pm 0,66$ & NS \\
\hline Amplitude distal & $14,8 \pm 5,68$ & $14,8 \pm 5,04$ & NS \\
\hline Amplitude proximal & $14,3 \pm 5,49$ & $13,6 \pm 5,62$ & NS \\
\hline Duração distal & $11,9 \pm 1,87$ & $12,5 \pm 2,48$ & NS \\
\hline Duração proximal & $11,8 \pm 1,58$ & $12,6 \pm 1,79$ & $*$ \\
\hline Velocidade de condução & $57,5 \pm 7,30$ & $56,5 \pm 5,68$ & NS \\
\hline \multicolumn{4}{|l|}{ Tibial posterior } \\
\hline Latência distal & $4,37 \pm 0,82$ & $4,54 \pm 1,21$ & NS \\
\hline Latência proximal & $12,5 \pm 1,65$ & $12,7 \pm 2,20$ & NS \\
\hline Amplitude distal & $21,5 \pm 7,50$ & $18,2 \pm 7,40$ & $*$ \\
\hline Amplitude proximal & $16,8 \pm 7,07$ & $15,7 \pm 6,63$ & NS \\
\hline Duração distal & $10,8 \pm 1,85$ & $11,4 \pm 1,83$ & NS \\
\hline Duração proximal & $12,3 \pm 1,99$ & $12,7 \pm 2,19$ & NS \\
\hline Velocidade de condução & $52,1 \pm 8,70$ & $53,2 \pm 11,9$ & NS \\
\hline \multicolumn{4}{|l|}{ Sural } \\
\hline Latência distal & $2,34 \pm 0,49$ & $2,40 \pm 0,47$ & NS \\
\hline Latência proximal & $5,03+0,74$ & $5,38 \pm 0,80$ & $*$ \\
\hline Amplitude distal & $27,2 \pm 8,83$ & $28,1 \pm 11,8$ & NS \\
\hline Amplitude proximal & $21,1 \pm 9,46$ & $17,7 \pm 6,18$ & NS \\
\hline Duração distal & $1,50 \pm 0,37$ & $1,50 \pm 0,37$ & NS \\
\hline Duração proximal & $1,66 \pm 0,32$ & $1,63 \pm 0,39$ & NS \\
\hline Velocidade de condução & $51,5 \pm 7,97$ & $52,5 \pm 9,58$ & NS \\
\hline
\end{tabular}

NS, não significante $(>0,05) ;{ }^{*}$, significante $(0,01-0,05)$ 


\section{DISCUSSÃO}

Alguns relatos de neuropatias periféricas no TMO associaram-se à toxicidade das drogas ${ }^{6,19-22}$, embora estudos não puderam relacionar causa-efeito.

No estudo de Wiznitzer e col. ${ }^{6}$, a lesão de nervos periféricos ocorreu em 14 de 57 pacientes: sete apresentaram herpes zoster dorsal, um manifestou mononeurite múltipla com envolvimento dos nervos peroneal e cutâneo lateral da coxa, relacionada à doença enxerto contra hospedeiro (DECH) severa. Os restantes envolveram nervos cranianos. Furlong e Gallucci ${ }^{4}$ detectaram 5 casos de neuropatia periférica entre 200 pacientes submetidos ao TMO. Curiosamente, descreveram maior prevalência no período pré-transplante. Embora com ressalvas relacionadas às limitações metodológicas, este estudo sugere relação temporal com a toxicidade do período de condicionamento. Na série de Graus e col. ${ }^{5}, 3$ de 425 pacientes apresentaram lesões de nervos periféricos. Em dois desses casos, a neuropatia ocorreu secundariamente a hemorragia intraneural durante o período de trombocitopenia severa com sangramento em múltiplos órgãos. O terceiro caso apresentou neuropatia de plexo braquial no dia +11 , coincidente com manifestação aguda de pele da $\mathrm{DECH}$, e foi definida como amiotrofia neurálgica. Neste estudo foram consideradas somente as alterações clinicamente significantes e excluídas as manifestações localizadas cutâneas do herpes zoster, o que justifica a baixa ocorrência de comprometimento periférico. Recente revisão no Serviço de TMO do Hospital de Clínicas da UFPR analisou 662 pacientes, dos quais 76 (11,5\%) apresentaram complicação do sistema nervoso periférico. $\mathrm{O}$ herpes zoster foi a complicação de maior incidência - ocorreu em 66 (10\%) dos casos. Foram descritos quatro casos de polineuropatia, dois de dermatopoliomiosite, dois de miotonia sem etiologia definida, um paciente com polirradiculopatia e mononeuropatia do túnel carpiano ${ }^{7}$.

Para auxílio diagnóstico das neuropatias periféricas, podemos utilizar métodos complementares como os testes quantitativos de sensibilidade. A sensibilidade vibratória, por exemplo, que é frequentemente pesquisada com o emprego de diapasão de baixa frequência, pode ser substituída por métodos quantitativos mais precisos como o palestesiômetro (vibrador elétrico) que mede o limiar de percepção vibratória fornecendo informações mais acuradas e tornando possível a detecção de alterações subclínicas iniciais. O uso deste teste quantitativo associado ao estudo da VCN pode aumentar a frequência diagnóstica. Outros exames complementares como o potencial evocado somatosensitivo, a análise do líquido cefalorraquidiano, os estudos da genética molecular e a biópsia do nervo sural também são úteis na investigação da neuropatia periférica. Suas aplicações atualmente não são rotineiras, sendo mais utilizadas em tipos específicos de neuropatias ${ }^{9}$.

Diversos fatores lesivos ao nervo devem ser lembrados na investigação desses pacientes. Condições determinantes de caquexia, tais como as doenças críticas crônicas, o câncer, o alcoolismo, a AIDS, as síndromes disabsortivas intestinais e os procedimentos cirúrgicos com redução de alças (malabsorção) são as principais causas de neuropatia periférica carencial ${ }^{14}$. Uma forma particular de neuropatia, a neuropatia dos pacientes críticos, também poderia ser considerada nos nossos pacientes, visto que ocorre em até $70 \%$ dos pacientes com sepsis ou falência de múltiplos órgãos ${ }^{15}$. Trata-se de polineuropatia axonal motora que envolve principalmente as fibras distais. A etiologia é frequentemente multifatorial, podendo estar relacionada à hipoalbuminemia. A incidência de neuropatia periférica parece aumentar na medida em que melhore o tratamento de suporte e o tempo de sobrevivência seja prolongado ${ }^{13}$. As leucemias aguda e crônica também podem comprometer o nervo periférico. São consideradas complicações raras, com estimativa inferior a 5\% mesmo quando combinadas com o envolvimento de nervos cranianos. A infiltração leucêmica é o principal mecanismo patogênico ${ }^{23}$. Outra forma de relação entre doença maligna e neuropatia periférica é a neuropatia paraneoplásica, que não tem sido descrita nesses tipos de neoplasias hematológicas ${ }^{23}$. A toxicidade dos nervos periféricos induzidos por droga é frequentemente relatada e, embora agentes de proteção estejam em estudo, o tratamento ainda se encontra na dependência do reconhecimento precoce dos sinais de toxicidade para permitir modificações pela redução de doses ou trocas terapêuticas? 
A proposta inicial deste estudo consistiu na busca ativa de neuropatia periférica durante os primeiros 90 dias pós-TMO (período coincidente com a necessidade de realização das principais avaliações gerais e com o aparecimento da doença enxerto contra hospedeiro aguda). A dificuldade de retorno do paciente trouxe remanejamento dessas avaliações que ocorreram durante a internação e alta do hospital, principalmente. Por restrições de ordem ética, realizamos o estudo da VCN apenas com eletrodos de superfície, não utilizando os de agulha, visto as condições de severa granulocitopenia dos pacientes, principalmente durante esse período do processo do TMO e o risco de bacteremias transitórias ${ }^{24}$.

As alterações neurológicas mais frequentes, no período observado, foram tremores de ação, de repouso, ou a combinação de ambos e coréia. A maioria dos pacientes submetidos a TMO sofre de náuseas e vômitos durante o período de toxicidade do condicionamento, que sem dúvida causam estresse e podem levar a distúrbios hidroeletrolíticos. O reconhecimento desta complicação culminou em estratégias de uso profilático de medicações anti-eméticas, isoladas ou em conjunto que, na dependência do agente, da dose e da sensibilidade individual, podem acarretar distúrbios extrapiramidais como os encontrados nos nossos pacientes. Eles foram inicialmente advertidos quanto ao uso da metroclopramida, droga auxiliar de escolha no suporte do condicionamento nesse Serviço, e após sua suspensão, houve melhora clínica. O achado de hiperreflexia profunda patelar e aquiléia no período pós-transplante não pode ser relacionado, podendo ser atribuído aos aspectos emocionais do paciente no período do exame.

Foram observadas diferenças na análise da glicemia e albuminemia. Acreditamos que essas alterações possam ocorrer no período em que realizamos esta pesquisa, visto que, devido à toxicidade induzida no condicionamento, os pacientes se tornam incapazes de alimentação oral e são nutridos parenteralmente, bem como mantidos sob soluções hipertônicas. Embora estatisticamente significativos, os valores variaram dentro da faixa de normalidade e não reproduziram alterações metabólicas que envolvessem o sistema nervoso central ou periférico.

O uso do palestesiômetro, no nosso estudo, não detectou alterações. O estudo da VCN demonstrou apenas alterações não específicas que isoladamente não foram consistentes para sugerir processo degenerativo. Consideramos as variabilidades relacionadas ao local do posicionamento de eletrodos como possíveis justificativas para a diferença verificada. Alguns sugerem que o uso de eletrodos grandes pode minimizar esta situação ${ }^{25,26}$. Ressaltamos que as técnicas de registros de condução utilizadas foram as rotineiras abrangendo, portanto, fibras grandes, cujos parâmetros podem estar normais em neuropatias de pequenas fibras.

Verificamos, com nossos resultados, que as drogas utilizadas neste Serviço não produzem toxicidade periférica no período recente pós-TMO (condicionamento e primeiro mês pós-TMO), fase em que o paciente usa uma combinação de inúmeras drogas potencialmente tóxicas e sob altas doses via parenteral. Reconhecemos que o estudo por biopsia poderia auxiliar na detecção de neuropatia de pequenas fibras. Estudos a longo prazo deveriam ser mantidos, para melhor estimativa desses resultados.

\section{REFERÊNCIAS}

1. Foucar K, Dyck FR. Interpretation of postchemotherapy and postransplantation bone marrow specimens. In Knowles DM (ed). Neoplastic hematopathology . Baltimore: Williams \& Wilkins 1992:1439-1455.

2. Pasquini R. Trasplante alogénico. In Borrasca et al. (eds). Enciclopedia iberoamericana de hematologia. España: Ed Univ Salamanca 1992:673-688.

3. Tabak DG. Transplante de medula. In Schwartsmann G. Oncologia clínica: princípios e prática. Porto Alegre: Artes Médicas 1991:171-180.

4. Furlong TG, Gallucci BB. Pattern of occurrence and clinical presentation of neurological complications in bone marrow transplant patients. Cancer Nursing 1994; 17:27-36.

5. Graus F, Saiz A, Sierra J, et al. Neurologic complications of autologous and allogeneic bone marrow transplantation in patients with leukemia. Neurology 1996;46:1004-1009.

6. Wiznitzer M, Packer RJ, August CS, Burkey ED. Neurological complications of bone marrow transplantation in childhood. Ann Neurol 1984; 16:569-576. 
7. Zétola VHF, Follador F, Teive HG, et al. Complicações neurológicas no transplante de medula óssea: análise de 662 casos (Abstr). Arq Neuropsiquiatr 1996; 54 (Suppl):O 91.

8. Kimura J. Nerve conduction studies and electromyography. In Dyck PJ et al. (eds). Peripheral neuropathy. 3 Ed. Philadelphia: WB Saunders, 1993:598-644.

9. McLeod JG. Investigation of peripheral neuropathy. J Neurol Neurosurg Psychiatry 1995;58:274-283.

10. Argov Z, Mastaglia FL. Drug induced peripheral neuropathies. Br Med J 1979;1:663-666.

11. Gilbert MR, Freimer ML. Neurotoxicity of cytotoxic agents. In Johnson RT, Griffin JW (eds). Current therapy in neurologic diseases. 4 Ed. St Louis: BC Decker, 1993:312-316.

12. MacDonald DR. Neurotoxicity of chemotherapeutic agents. In Perry MC (ed). The chemotherapy source book. Baltimore: Williams \& Wilkins, 1992:666-679.

13. Latronico N, Fenzi F, Recupero D, et al. Critical illness myopathy and neuropathy. Lancet 1996;1:1579-1582.

14. Windebank AJ. Polyneuropathy due to nutricional deficiency and alcoholism. In Dyck PJ et al. (eds). Peripheral neuropathy. 3 Ed. Philadelphia: WB Saunders, 1993:1311-1321.

15. Witt NJ, Zochodne DW, Bolton CF, et al. Peripheral nerve function in sepsis and multiple organ failure. Chest 1991;99:176-184.

16. Werneck LC, Mulinari AS, Laffite A, Kesikowski LB. Polineuropatia urêmica: estudo clínico-eletroneurográfico de 33 casos. Arq Neuropsiquiatr 1979;37:356-372.

17. Werneck LC, Mulinari AS, Laffite A, Kesikowski LB. Polineuropatia urêmica: estudo do limiar de percepção vibratória em 19 pacientes. Arq Neuropsiquiatr 1984;42:215-220.

18. Steiness IB. Vibratory perception in normal subjects. Act Med Scand 1957;158:315-325.

19. Amato AA, Barohn RJ, Sahenk Z, Tutschka PJ, Mendell JR. Polyneuropathy complicating bone marrow and solid organ transplantation. Neurology 1993;43:1513-1518

20. Greenspan A, Deeg HJ, Cottler-Fox M, Sirdofski M, Spitzer TR, Kattah J. Incapacitating peripheral neuropathy as a manifestation of chronic graft-versus-host disease. Bone Marrow Transplant 1990;5:349-352.

21. Maguire H, August C, Sladky J. Chronic inflammatory demyelinating polyneuropathy: a previosly unreported complication of fone marrow transplantation. Neurology 1989;39(Suppl 1):410.

22. Vogelsang GB, Farmer ER, Hess AD, et al. Thalidomide for the treatment of chronic graft-versus-host disease. N Engl J Med 1992;326:1055-1058.

23. McLeod JG. Peripheral neuropathy associated with lymphomas, leukemias, and polycythemia vera. In Dyck PJ et al. (eds). Peripheral neuropathy, 3 Ed. Philadelphia: WB Saunders, 1993:159-198.

24. Daube JR. The art of needle electromyography. Annual Seminars Vol XI Neuromuscular Disease. San Francisco, 1966; 377:01-26.

25. Tjon-A-Tsien AML, Lemkes HHPJ, van der Kamp-Huyts AJC, van Dijk JG. Large electrodes improve nerve conduction repeatability in controls as well as in patients with diabetic neuropathy. Muscle Nerve 1996;19:689-695.

26. van Dijk JG, Tjon-A-Tsien AML, van der Kamp W. CMAP variability as a function of electrode site and size. Muscle Nerve 1995;18:68-73. 